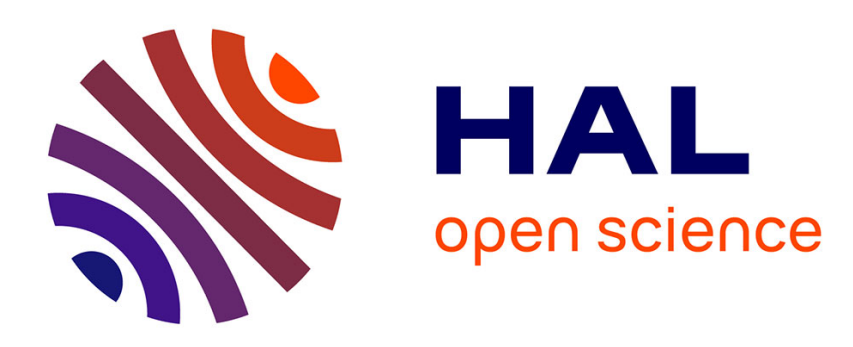

\title{
Fano manifolds with nef tangent bundles are weakly almost Kähler-Einstein
}

Jean-Pierre Demailly

\section{To cite this version:}

Jean-Pierre Demailly. Fano manifolds with nef tangent bundles are weakly almost Kähler-Einstein. 2018. hal-01660131v2

\section{HAL Id: hal-01660131 \\ https://hal.science/hal-01660131v2}

Preprint submitted on 4 Feb 2018

HAL is a multi-disciplinary open access archive for the deposit and dissemination of scientific research documents, whether they are published or not. The documents may come from teaching and research institutions in France or abroad, or from public or private research centers.
L'archive ouverte pluridisciplinaire HAL, est destinée au dépôt et à la diffusion de documents scientifiques de niveau recherche, publiés ou non, émanant des établissements d'enseignement et de recherche français ou étrangers, des laboratoires publics ou privés. 


\title{
FANO MANIFOLDS WITH NEF TANGENT BUNDLES ARE WEAKLY ALMOST KÄHLER-EINSTEIN
}

\author{
JEAN-PIERRE DEMAILLY
}

\begin{abstract}
The goal of this short note is to point out that every Fano manifold with a nef tangent bundle possesses an almost Kähler-Einstein metric, in a weak sense. The technique relies on a regularization theorem for closed positive $(1,1)$-currents. We also discuss related semistability questions and Chern inequalities.
\end{abstract}

dedicated to Professor Ngaiming Mok on the occasion of his sixtieth birthday

\section{INTRODUCTION}

Recall that a holomorphic vector bundle $E$ on a projective manifold $X$ is said to be numerically effective (nef) if the line bundle $\mathcal{O}_{\mathbb{P}(E)}(1)$ is nef on $Y=\mathbb{P}(E)$. Clearly, every homogeneous projective manifold $X$ has its tangent bundle $T_{X}$ generated by sections, so $T_{X}$ is nef; morever, by [DPS94, every compact Kähler manifold with $T_{X}$ nef admits a finite étale cover $\tilde{X}$ that is a locally trivial fibration over its Albanese torus, and the fibers are themselves Fano manifolds $F$ with $T_{F}$ nef. Hence, the classification problem is essentially reduced to the case when $X$ is a Fano manifold with $T_{X}$ nef. In this direction, Campana and Peternell CP91 conjectured in 1991 that Fano manifolds with nef tangent bundles are rational homogeneous manifolds $G / P$, namely quotients of linear algebraic groups by parabolic subgroups. Although this can be checked up to dimension 3 by inspecting the classification of Fano 3-folds by Manin-Iskovskii and Mukai, very little is known in higher dimensions. It would be tempting to use the theory of VMRT's developed by J.M. Hwang and N. Mok ([Hwa01], Mok08]), since the expected homogeneity property should be reflected in the geometry of rational curves. Even then, the difficulties to be solved remain formidable; see e.g. Mok02 and also [MOSWW] for a recent account of the problem.

On the other hand, every rational homogeneous manifold $X=G / P$ carries a Kähler metric that is invariant by a compact real form $G^{\mathbb{R}}$ of $G$ (cf. [AP86]), and the corresponding Ricci curvature form (i.e. the curvature of $-K_{G / P}$ ) is then a Kähler-Einstein metric. A stronger condition than nefness of $T_{X}$ is the existence of a Kähler metric on $X$ whose holomorphic bisectional curvature is nonnegative. N. Mok [Mok88] characterized those manifolds, they are exactly the products of hermitian symmetric spaces of compact type by flat compact complex tori $\mathbb{C}^{q} / \Lambda$ and projective spaces $\mathbb{P}^{n_{j}}$ with a Kähler metric of nonnegative holomorphic bisectional curvature. However, hermitian symmetric spaces of compact type are a smaller class than rational homogeneous manifolds (for instance, they do not include complete flag

2010 Mathematics Subject Classification. 14J45, 14M17, 32C30, 32Q10, 32Q20.

Key words and phrases. Fano manifold, numerically effective vector bundle, rational homogeneous manifold, Campana-Peternell conjecture, Kähler-Einstein metric, closed positive current, regularization of currents, Schauder fixed point theorem. 
manifolds), thus one cannot expect these Kähler-Einstein metrics to have a nonnegative bisectional curvature in general.

It is nevertheless a natural related question to investigate whether every Fano manifold $X$ with nef tangent bundle actually possesses a Kähler-Einstein metric. Our main observation is the following (much) weaker statement, whose proof is based on regularization techniques for closed positive currents [Dem92], Dem99]. We denote here by $\mathrm{PC}^{1,1}(X)$ the cone of positive currents of bidegree $(1,1)$ on $X$ and put $n=\operatorname{dim}_{\mathbb{C}} X$. Though it is a convex set of infinite dimension, the intersection $\mathrm{PC}^{1,1}(X) \cap c_{1}(X)$ is compact and metrizable for the weak topology (see $\S 2$ ), and therefore it carries a unique uniform structure that can be defined by any compatible metric.

Theorem 1.1. Let $X$ be a Fano manifold such that the tangent bundle $T_{X}$ is nef. Then

(i) there exists a family of smoothing operators $\left(J_{\varepsilon}\right)_{\varepsilon \in] 0,1]}$ that map every closed positive current $T \in \mathrm{PC}^{1,1}(X) \cap c_{1}(X)$ to a smooth closed positive definite $(1,1)$-form

$$
\alpha_{\varepsilon}=J_{\varepsilon}(T) \in c_{1}(X),
$$

in such a way that $J_{\varepsilon}(T)$ converges weakly to $T$ as $\varepsilon \rightarrow 0$ (uniformly for all $T$ ), and $T \mapsto J_{\varepsilon}(T)$ is continuous with respect to the weak topology of currents and the strong topology of $C^{\infty}$ convergence on smooth $(1,1)$-forms;

(ii) for every $\varepsilon \in] 0,1]$, there exists a Kähler metric $\omega_{\varepsilon}$ on $X$ such that $\operatorname{Ricci}\left(\omega_{\varepsilon}\right)=J_{\varepsilon}\left(\omega_{\varepsilon}\right)$, in other words $\omega_{\varepsilon}$ is "weakly almost Kähler-Einstein".

The construction of $J_{\varepsilon}$ that we have is unfortunately not very explicit, and we do not even know if $J_{\varepsilon}$ can be constructed as a natural linear (say convolution-like) operator. The proof of (ii) is based on the use of the Schauder fixed point theorem. Let us denote by

$$
0<\rho_{1, \varepsilon}(x) \leq \ldots \leq \rho_{n, \varepsilon}(x)
$$

the eigenvalues of $\operatorname{Ricci}\left(\omega_{\varepsilon}\right)$ with respect to $\omega_{\varepsilon}$ at each point $x \in X$, and $\zeta_{1, \varepsilon}, \ldots, \zeta_{n, \varepsilon} \in T_{X, x}$ a corresponding orthonormal family of eigenvectors with respect to $\omega_{\varepsilon}$. We know that

$$
\int_{X} \sum_{j=1}^{n} \rho_{j, \varepsilon} \omega_{\varepsilon}^{n}=n \int_{X} \operatorname{Ricci}\left(\omega_{\varepsilon}\right) \wedge \omega_{\varepsilon}^{n-1}=n c_{1}(X)^{n},
$$

in particular the left-hand side is bounded. Also, as $\operatorname{Ricci}\left(\omega_{\varepsilon}\right)-\omega_{\varepsilon}=J_{\varepsilon}\left(\omega_{\varepsilon}\right)-\omega_{\varepsilon}$ converges weakly to 0 , we know that

$$
\int_{X} \sum_{j=1}^{n}\left(\rho_{j, \varepsilon}-1\right) \zeta_{1, \varepsilon}^{*} \wedge \overline{\zeta_{1, \varepsilon}^{*}} \wedge u
$$

converges to 0 for every smooth $(n-1, n-1)$-form $u$ on $X$, hence the eigenvalues $\rho_{j, \varepsilon}$ "converge weakly to 1 " in the sense that $\sum_{j=1}^{n}\left(\rho_{j, \varepsilon}-1\right) \zeta_{1, \varepsilon}^{*} \wedge \overline{\zeta_{1, \varepsilon}^{*}}$ converges weakly to 0 in the space of smooth $(1,1)$-forms. Therefore, it does not seem too unrealistic to expect that the family $\left(\omega_{\varepsilon}\right)$ is well behaved in the following sense.

Definition 1.2. We say the family $\left(\omega_{\varepsilon}\right)$ of weak almost Kähler-Einstein metrics is well behaved in $L^{p}$ norm if

$(* *)^{p}$

$$
\int_{X}\left(\rho_{n, \varepsilon}-\rho_{1, \varepsilon}\right)^{p} \omega_{\varepsilon}^{n}
$$

converges to 0 as $\varepsilon$ tends to 0 . 
Standard curvature inequalities then yield the following result.

Theorem 1.3. Assume that $X$ is Fano with $T_{X}$ nef, and possesses a family of weak almost Kähler-Einstein metrics that is well behaved in $L^{p}$ norm. Then

(i) if $p \geq 1, T_{X}$ is $c_{1}(X)$-semistable;

(ii) if $p \geq 2$, the Guggenheimer-Yau-Bogomolov-Miyaoka Chern class inequality

$$
\left[n c_{1}(X)^{2}-(2 n+2) c_{2}(X)\right] \cdot c_{1}(X)^{n-2} \leq 0
$$

is satisfied.

The author is indebted to the referee and to Yury Ustinovskiy for pointing out some issues in an earlier version of this work. He expresses warm thanks to Ngaiming Mok, Jun-Muk Hwang, Thomas Peternell, Yum-Tong Siu and Shing-Tung Yau for inspiring discussions or viewpoints around these questions in the last 25 years (or more!).

\section{Proofs}

Existence of REgularization operators in 1.1(i). Let us fix once for all a Kähler metric $\beta \in c_{1}(X)$. It is well known that $K=\mathrm{PC}^{1,1}(X) \cap c_{1}(X)$ is compact for the weak topology of currents; this comes from the fact that currents $T \in K$ have a uniformly bounded mass

$$
\int_{X} T \wedge \beta^{n-1}=c_{1}(X)^{n}
$$

We also know from [Dem92, Dem99] that every such current $T$ admits a family of regularizations $T_{\varepsilon} \in C^{\infty}(X) \cap c_{1}(X)$ converging weakly to $T$, such that $T_{\varepsilon} \geq-\varepsilon \beta$. Here, the fact that we can achieve an arbitrary small lower bound uses in an essential way the assumption that $T_{X}$ is nef. After replacing $T_{\varepsilon}$ by $T_{\varepsilon}^{\prime}=(1-\varepsilon) T_{\varepsilon}+\varepsilon \beta \geq \varepsilon^{2} \beta$, we can assume that $T_{\varepsilon}$ is a Kähler form. An important issue is that we want to produce a continuous operator $J_{\varepsilon}(T)=T_{\varepsilon}$ defined on the weakly compact set $K$. This is clearly the case when the regularization is produced by convolution, as is done Dem94, but in that case one needs a more demanding condition on $T_{X}$, e.g. that $T_{X}$ possesses a hermitian metric with nonnegative bisectional curvature (i.e. that $T_{X}$ is Griffiths semipositive). However, the existence of a continuous global operator $J_{\varepsilon}$ is an easy consequence of the convexity of $K$. In fact, the weak topology of $K$ is induced by the $L^{2}$ topology on the space of potentials $\varphi$ when writing $T=\beta+d d^{c} \varphi$ (and taking the quotient by constants) - it is also induced by the $L^{p}$ topology for any $p \in\left[1, \infty\left[\right.\right.$, but $L^{2}$ has the advantage of being a Hilbert space topology. By compactness, for every $\delta \in] 0,1]$ we can then find finitely many currents $\left(T_{j}\right)_{1 \leq j \leq N(\delta)}$ such that the Hilbert balls $B\left(T_{j}, \delta\right)$ cover $K$. Let $K_{\delta}$ be the convex hull of the family $\left(T_{j}\right)_{1 \leq j \leq N(\delta)}$ and let $p_{\delta}: K \rightarrow K_{\delta}$ be induced by the (nonlinear) Hilbert projection $L^{2} / \mathbb{R} \rightarrow K_{\delta}$. Since $K_{\delta}$ is finite dimensional and is a finite union of simplices, we can take regularizations $T_{j, \varepsilon}$ of the vertices $T_{j}$ and construct a piecewise linear operator $\tilde{J}_{\delta, \varepsilon}: K_{\delta} \rightarrow K \cap C^{\infty}(X)^{+}$to Kähler forms, simply by taking linear combinations of the $T_{j, \varepsilon}$ 's. Then $\tilde{J}_{\delta, \varepsilon} \circ p_{\delta}(T)$ is the continuous regularization operator we need. The uniform weak convergence to $T$ is guaranteed if we take $J_{\varepsilon}:=\tilde{J}_{\delta(\varepsilon), \varepsilon}$ with $\varepsilon \ll \delta(\varepsilon) \rightarrow 0$, e.g. with a step function $\varepsilon \mapsto \delta(\varepsilon)$ that converges slowly to 0 compared to $\varepsilon$. These operators have the drawback of being non explicit and a priori non linear. 
Question 2.1. Is it possible to construct a linear regularization operator $J_{\varepsilon}$ with the same properties as above, e.g. by means of a convolution process?

In fact, such a "linear" regularization operator $J_{\varepsilon}$ is constructed in Dem94 by putting $T=\beta+d d^{c} \varphi$ and $J_{\varepsilon}(T)=\beta+d d^{c} \varphi_{\varepsilon}$ where

$$
\varphi_{\varepsilon}(z)=\int_{\zeta \in T_{X, z}} \varphi\left(\operatorname{exph}_{z}(\zeta)\right) \chi\left(|\zeta|_{h}^{2} / \varepsilon^{2}\right) d V_{h}(\zeta)
$$

for a suitable hermitian metrics $h$ on $T_{X}$, taking exph to be the holomorphic part of the exponential map associated with the Chern connection $\nabla_{h}$. However, the positivity of $J_{\varepsilon}(T)$ is in general not preserved, unless one assumes that $\left(T_{X}, h\right)$ is (say) Griffiths semipositive. As the relation between nefness and Griffiths semipositivity is not yet elucidated, one would perhaps need to extend the above formula to the case of Finsler metrics. This is eventually possible by applying some of the techniques of [Dem99] to produce suitable dual Finsler metrics on $T_{X}$ (notice that any assumption that $T_{X} \otimes L$ is ample for some $\mathbb{Q}$-line bundle $L$ translates into the existence of a strictly plurisubharmonic Finsler metric on the total space of $\left(T_{X} \otimes L\right)^{*} \backslash\{0\}$, so one needs to dualize).

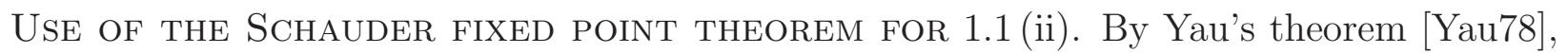
for every closed $(1,1)$-form $\rho \in c_{1}(X)$, there exists a unique Kähler metric $\gamma(\rho) \in c_{1}(X)$ such that $\operatorname{Ricci}(\gamma(\rho))=\rho$. Moreover, by the regularity theory of nonlinear elliptic operators, the map $\rho \mapsto \gamma(\rho)$ is continuous in $C^{\infty}$ topology. We consider the composition

$$
\gamma \circ J_{\varepsilon}: K \mapsto K \cap C^{\infty}(X)^{+} \rightarrow K \cap C^{\infty}(X)^{+} \subset K, \quad T \mapsto \rho=J_{\varepsilon}(T) \mapsto \gamma(\rho) .
$$

Since $J_{\varepsilon}$ is continuous from the weak topology to the strong $C^{\infty}$ topology, we infer that $\gamma \circ J_{\varepsilon}$ is continuous on $K$ in the weak topology. Now, $K$ is convex and weakly compact, therefore $\gamma \circ J_{\varepsilon}$ must have a fixed point $T=\omega_{\varepsilon}$ by the theorem of Schauder. By construction $\omega_{\varepsilon}$ must be a Kähler metric in $c_{1}(X)$, since $\gamma \circ J_{\varepsilon}$ maps $K$ into the space of Kähler metrics contained in $c_{1}(X)$. This proves Theorem 1.1.

Semistability of $T_{X}(1.3(\mathrm{i}))$. Let $\mathcal{F} \subset \mathcal{O}\left(T_{X}\right)$ be a coherent subsheaf such that $\mathcal{O}\left(T_{X}\right) / \mathcal{F}$ is torsion free. We can view $\mathcal{F}$ as a holomorphic subbundle of $T_{X}$ outside of an algebraic subset of codimension 2 in $X$. The Chern curvature tensor $\Theta_{T_{X}, \omega_{\varepsilon}}=\frac{i}{2 \pi} \nabla^{2}$ satisfies the Hermite-Einstein condition

$$
\Theta_{T_{X}, \omega_{\varepsilon}} \wedge \omega_{\varepsilon}^{n-1}=\frac{1}{n} \rho_{\varepsilon} \omega_{\varepsilon}^{n}
$$

where $\rho_{\varepsilon} \in C^{\infty}\left(X, \operatorname{End}\left(T_{X}\right)\right)$ is the Ricci operator (with the same eigenvalues $\rho_{j, \varepsilon}$ as $\operatorname{Ricci}\left(\omega_{\varepsilon}\right)$ ). It is well known that the curvature of a subbundle is always bounded above by the restriction of the full curvature tensor, i.e. $\Theta_{\mathcal{F}, \omega_{\varepsilon}} \leq \Theta_{T_{X}, \omega_{\varepsilon} \mid \mathcal{F}}$ (say, in the sense of Griffiths positivity, viewing the curvature tensors as hermitian forms on $T_{X} \otimes \mathcal{F}$ ). By taking the trace with respect to $\omega_{\varepsilon}$, we get

$$
\Theta_{\mathcal{F}, \omega_{\varepsilon}} \wedge \omega_{\varepsilon}^{n-1} \leq \frac{1}{n} \rho_{\varepsilon \mid \mathcal{F}} \omega_{\varepsilon}^{n}
$$

By the minimax principle, if $r=\operatorname{rank} \mathcal{F}$, the eigenvalues of $\rho_{\varepsilon \mid \mathcal{F}}$ are bounded above by

$$
\rho_{n-r+1, \varepsilon} \leq \ldots \leq \rho_{n, \varepsilon}
$$


FANO MANIFOLDS WITH NEF TANGENT BUNDLES ARE WEAKLY ALMOST KÄHLER-EINSTEIN 5

hence

$$
\int_{X} c_{1}(\mathcal{F}) \wedge \omega_{\varepsilon}^{n-1}=\int_{X} \operatorname{tr}_{\mathcal{F}} \Theta_{\mathcal{F}, \omega_{\varepsilon}} \wedge \omega_{\varepsilon}^{n-1} \leq \frac{1}{n} \int_{X} \sum_{j=1}^{r} \rho_{n-r+j, \varepsilon} \omega_{\varepsilon}^{n} .
$$

The left hand side is unchanged if we replace $\omega_{\varepsilon}$ by $\beta \in c_{1}(X)$, and our assumption $(* *)^{p}$ for $p=1$ (cf. Definition 1.2) implies

$$
\int_{X} c_{1}(\mathcal{F}) \wedge \beta^{n-1} \leq \lim _{\varepsilon \rightarrow 0} \frac{1}{n} \int_{X} \sum_{j=1}^{r} \rho_{n-r+j, \varepsilon} \omega_{\varepsilon}^{n}=\lim _{\varepsilon \rightarrow 0} \frac{r}{n^{2}} \int_{X} \sum_{j=1}^{n} \rho_{j, \varepsilon} \omega_{\varepsilon}^{n}=\frac{r}{n} \int_{X} c_{1}(X) \wedge \beta^{n-1}
$$

by $(*)$. This means that $T_{X}$ is $c_{1}(X)$-semistable.

Chern Class inequality (1.3)(ii)). Let us write

$$
\Theta_{T_{X}, \omega_{\varepsilon}}=\left(\theta_{\alpha \beta}\right)_{1 \leq \alpha, \beta \leq n}=\left(\sum_{j, k} \theta_{\alpha \beta j k} d z_{j} \wedge d \bar{z}_{k}\right)_{1 \leq \alpha, \beta \leq n}
$$

as an $n \times n$ matrix of $(1,1)$-forms with respect to an orthonormal frame of $T_{X}$ that diagonalizes the Ricci operator $\rho_{\varepsilon}$ (with respect to the metric $\omega_{\varepsilon}$ ). A standard calculation yields

$$
\begin{aligned}
& 2\left[n c_{1}(X)_{h}^{2}-(2 n+2) c_{2}(X)\right] \wedge \frac{\omega_{\varepsilon}^{n-2}}{(n-2) !} \\
&=\left(-\sum_{\alpha \neq \beta, j \neq k}\left|\theta_{\alpha \alpha j k}-\theta_{\beta \beta j k}\right|^{2}-(n+1) \sum_{\alpha, \beta, j, k, \text { pairwise } \neq}\left|\theta_{\alpha \beta j k}\right|^{2}\right. \\
&-(4 n+8) \sum_{\alpha \neq j<k \neq \alpha}\left|\theta_{\alpha \alpha j k}\right|^{2}-4 \sum_{\alpha \neq j}\left|\theta_{\alpha \alpha \alpha j}\right|^{2} \\
&-\sum_{\alpha \neq \beta \neq j \neq \alpha}\left|\theta_{\alpha \alpha j j}-\theta_{\beta \beta j j}\right|^{2}-\sum_{\alpha \neq \beta}\left|\theta_{\alpha \alpha \alpha \alpha}-2 \theta_{\alpha \alpha \beta \beta}\right|^{2} \\
&\left.+n \sum_{\alpha} \rho_{\alpha, \varepsilon}^{2}-\left(\sum_{\alpha} \rho_{\alpha, \varepsilon}\right)^{2}\right) \omega_{\varepsilon}^{n}
\end{aligned}
$$

where all terms in the summation are nonpositive except the ones involving the Ricci eigenvalues $\rho_{\alpha, \varepsilon}$. However, the assumption $(* *)^{p}$ for $p \geq 2$ implies that the integral of the difference appearing in the last line converges to 0 as $\varepsilon \rightarrow 0$. Theorem 1.3 is proved.

Our results lead to the following interesting question.

Question 2.2. Assuming that a "sufficiently good" family of regularization operators $J_{\varepsilon}$ is used, can one infer that the resulting family $\left(\omega_{\varepsilon}\right)$ of fixed points such that $\operatorname{Ricci}\left(\omega_{\varepsilon}\right)=J_{\varepsilon}\left(\omega_{\varepsilon}\right)$ (or some subsequence) is well behaved in $L^{1}$, resp. $L^{2}$ norm?

Remark 2.3. A strategy to attack the Campana-Peternell conjecture could be as follows. The first step would be to prove that $H^{0}\left(X, T_{X}\right) \neq 0$ (if $\operatorname{dim} X>0$ ). Assume therefore $H^{0}\left(X, T_{X}\right)=0$ and try to reach a contradiction. It is well known in that case that there are much fewer obstructions to the existence of Kähler-Einstein metrics, for instance one has the Bando-Mabuchi uniqueness theorem [BM85] and (obviously) the vanishing of the classical Futaki invariant [Fut83]; hence we could expect our family of weakly almost Kähler-Einstein metrics to converge to a genuine Kähler-Einstein metric. But then the resulting known Chern class inequalities (the ones of Theorem 1.3 (ii) combined with the Fulton-Lazarsfeld 
inequalities [FL83], [DPS94]) might help to contradict $H^{0}\left(X, T_{X}\right)=0$, e.g. by the RiemannRoch formula or by ad hoc nonvanishing theorems such as the generalized hard Lefschetz theorem [DPS01]. Now, the existence of holomorphic vector fields on $X$ implies that $X$ has a non trivial group of automorphisms $H=\operatorname{Aut}(X)$, and one could then try to apply induction on dimension on a suitable desingularization $Y$ of $X / / H$, if $Y$ is not reduced to a point and one can achieve $Y$ to have $T_{Y}$ nef.

\section{REFERENCES}

[AP86] Alekseevskit, D. V., Perelomov, A.M., Invariant Kähler-Einstein metrics on compact homogeneous spaces, Funktsional. Anal. i Prilozhen. 20(3) (1986), 1-16; English version: Functional Analysis and Its Applications 20(3), (1986) 171-182.

[BM85] Bando, S., Mabuchi, T., Uniqueness of Einstein Kähler metrics modulo connected group actions, Algebraic geometry, Sendai, 1985, Adv. Stud. Pure Math., 10, North-Holland, Amsterdam, 1987, $11-40$.

[CP91] Campana, F., Peternell, Th., Projective manifolds whose tangent bundles are numerically effective, Math. Ann. 289(1) (1991), 169-187.

[CP93] Campana, F., Peternell, Th., 4-folds with numerically effective tangent bundles and second Betti numbers greater than one, Manuscripta Math. 79(3-4) (1993), 225-238.

[Dem92] Demailly, J.-P., Regularization of closed positive currents and Intersection Theory, J. Alg. Geom. 1 (1992) 361-409.

[Dem94] Demailly, J.-P., Regularization of closed positive currents of type $(1,1)$ by the flow of a Chern connection, Actes du Colloque en lhonneur de P. Dolbeault (Juin 1992), edited by H. Skoda and J.M. Trépreau, Aspects of Mathematics, Vol. E26, Vieweg (1994), 105-126.

[Dem99] Demailly, J.-P., Pseudoconvex-concave duality and regularization of currents, Several Complex Variables, MSRI publications, Volume 37, Cambridge Univ. Press (1999), 233-271.

[DPS94] Demailly, J.-P., Peternell, Th., Schneider, M., Compact complex manifolds with numerically effective tangent bundles, J. Algebraic Geom. 3(2) (1994), 295-345.

[DPS01] Demailly, J.-P., Peternell, Th., Schneider, M., Pseudo-effective line bundles on compact Kähler manifolds International Journal of Math. 6 (2001) 689-741.

[Fl83] Fulton, W., Lazarsfeld, R., Positive polynomials for ample vector bundles, Ann. Math. 118 (1983) 35-60.

[Fut83] Futaki, A., An obstruction to the existence of Einstein Kähler metrics, Invent. Math. 73 (1983), 437-443.

[Hwa01] Hwang, J.M., Geometry of minimal rational curves on Fano manifolds, In: School on Vanishing Theorems and Effective Results in Algebraic Geometry (Trieste, 2000), volume 6 of ICTP Lect. Notes, Abdus Salam Int. Cent. Theoret. Phys., Trieste, 2001, 335-393.

[Mok08] Mok, N., Geometric structures on uniruled projective manifolds defined by their varieties of minimal rational tangents, Astérisque 322 (2008) 151-205, in: Géométrie différentielle, physique mathématique, mathématiques et société II.

[Mok88] MoK, N., The uniformization theorem for compact Kähler manifolds of nonnegative holomorphic bisectional curvature, J. Differential Geom. 27(2) (1988), 179-214.

[Mok02] Mok, N., On Fano manifolds with nef tangent bundles admitting 1-dimensional varieties of minimal rational tangents, Trans. Amer. Math. Soc. 354(7) (2002) 2639-2658.

[MOSWW] Muñoz, R., Occhetta, G., Solá Conde, L.E., Watanable, K., Wiśniewski, J.A., A survey on the Campana-Peternell conjecture, Rend. Istit. Mat. Univ. Trieste 47 (2015), 127-185.

[Yau78] YAu, S.T., On the Ricci curvature of a complex Kähler manifold and the complex Monge-Ampère equation I, Comm. Pure and Appl. Math. 31 (1978), 339-411.

Jean-Pierre Demailly

Université Grenoble Alpes, Institut Fourier, F-38000 Grenoble, France

CNRS, Institut Fourier, F-38000 Grenoble, France

e-mail: jean-pierre.demailly@univ-grenoble-alpes.fr 